\title{
Quality of Care for Patients with Acute Myocardial Infarction (AMI) in Pakistan: A Retrospective Study
}

\author{
Shazia Rehman ${ }^{1}, \mathrm{Xi} \mathrm{Li}^{1}$, Chao Wang ${ }^{1}$, Muhammad Ikram ${ }^{2}{ }^{-0}$, Erum Rehman ${ }^{3}$ \\ and Meina Liu $1, *(\mathbb{D})$ \\ 1 Department of Biostatistics, School of Public Health, Harbin Medical University, No.157 Baojian Road, \\ Harbin 150081, China; s.rehmaan@outlook.com (S.R.); lixi_094@163.com (X.L.); \\ wangchao_ln1219@163.com (C.W.) \\ 2 College of Economics and management, Nanjing University of Aeronautics and Astronautics, 29 Jiangsu \\ Avenue, Nanjing 211106, China; mikram@nuaa.edu.cn \\ 3 School of Mathematics and Statistics, Southwest University of Finance and Economics, \\ Chengdu 610074, China; 117020208901@smail.swufe.edu.cn \\ * Correspondence: liumeina@ems.hrbmu.edu.cn
}

Received: 15 August 2019; Accepted: 12 October 2019; Published: 14 October 2019

\begin{abstract}
A wide variation exists in the practice patterns of acute myocardial infarction (AMI) care worldwide, leading to differences in clinical outcomes. This study aims to evaluate the quality of process care and its impact on in-hospital outcomes among AMI patients in Pakistan, as no such study has been conducted in Pakistan thus far based upon recommended guidelines. We investigated a sample of 2663 AMI patients across 11 territory hospitals in Punjab province of Lahore, Faisalabad, Multan, Rawalpindi, and Islamabad from January 1, 2016 to December 31, 2017, with an in-hospital mortality rate of 8.6\%. We calculated compliance rates of quality indicators (QIs) for all eligible patients. The association between process care and in-hospital outcome was assessed using hierarchical generalized linear model that adjusted for patient and hospital characteristics. In addition, we examined the effect of patient composite scores on clinical outcomes. Aspirin $(73.08 \%)$ and clopidogrel $(67.86 \%)$ indicated relatively better conformance than other QIs. The percutaneous coronary intervention also showed significantly low adherence. All QIs showed no significant association with in-hospital mortality. In contrast, 4 out of 8 QIs were observed positively correlated with in-hospital length of stay (LOS). The overall patient composite score was found to be statistically significant with in-hospital LOS. The assessment of quality of care showed low adherence to clinical care recommendations, and increased adherence was associated with longer in-hospital LOS among AMI patients. Evaluation of valid QIs for AMI treatment and their impact on in-hospital outcomes is an important tool for improving health care delivery in the overall AMI population in Pakistan. Low adherence to performance measures strongly compel to focus on guideline-based tools for AMI in Pakistan.
\end{abstract}

Keywords: acute myocardial infarction; quality of care; in-hospital outcomes; compliance measurement; composite score

\section{Introduction}

Despite attempts by professional societies to standardize acute myocardial infarction (AMI) care through the guidelines, substantial variation exists in the practice patterns for the management of AMI in hospitals around the world. In the era of accountability in the therapeutic field, evaluation of quality of care plays a vital role and has turned into an imperative tool for health specialists and experts [1]. However, assessing the quality of care in patients admitted for acute myocardial infarction (AMI) is challenging because it is a complex notion that is based on a wide broad variety of factors not restricted 
to positive clinical results only [2]. Therefore, evaluation of the quality of care by quality indicators (QIs) has become a standard, global practice that captures the detailed outcome of treatment programs accurately [3-6]. Several countries and territories have introduced a number of QIs to evaluate and assess the quality of health care in AMI patients [7-12]. In a few western countries, the implementation of QIs for AMI patients has delivered significant outcomes and has likewise shown their capacity to identify fluctuations in care across hospitals $[2,3,6,13]$.

AMI is among the leading causes of death and disability worldwide. It has a strong significance in accomplishing rapid revascularization due to on-time diagnosis and treatment which largely affects survival [14]. Accurate burden assessment of patients with AMI in low- and middle-income countries is mostly unavailable; however, crude estimates indicate that the burden of AMI has been steadily increasing over the last decades in low- and middle-income countries [15]. Cardiovascular risk factors for ischemic heart disease and AMI are on the rise in Pakistan and a leading cause of morbidity and mortality [16]. However, Pakistan is spending less than $1 \%$ of the gross domestic product (GDP) on public health for the past few decades, while the World Health Organization (WHO) benchmark of health expenditure is at least $6 \%$ of the GDP [17].

Although individual QIs are useful to measure the outcomes for improvement activities, questions remain concerning how to use the available data to assess and quantify progress made in quality improvement. Composite measures aggregate individual QIs into a summary of performance across multiple dimensions of care, which allows an overall representation of healthcare quality provided and provides a more understandable format. To date, there has been no investigation done in context of Pakistan for assessing the quality of AMI care based upon the recommended guidelines or the association between the QIs and in-hospital outcomes. Previous studies [18] have emphasized the association between process of care measures and in-hospital outcome, but have been limited ascertainment of in-hospital events, which can be substantially biased by in-hospital length of stay. Thus, the degree to which process measure performance conveys meaningful information about in-hospital mortality remains unclear. To address the above-mentioned needs, we aimed to estimate the compliance rate of QIs, and examine the association between quality indicators care and in-hospital outcomes among ST Segment Elevation Myocardial Infarction (STEMI) and Non-ST Segment Elevation Myocardial Infarction (NSTEMI) patients, including individual QIs and patient composite score. Identifying valid QIs for AMI treatment and their impact on in-hospital outcomes, is an important tool for improving the quality of health care.

To the best of our insight, this study is the first of its kind done in the Pakistani population to assess the relationship between quality care and in-hospital outcomes among AMI patients. The primary quality of our investigation is that it provides the most recent statistics for evaluating quality of care delivery for patients with AMI in Pakistan. Also, it provides an estimation of composite score from the patient dimension using quality indicators.

\section{Methods}

\subsection{Data Source and Study Population}

A retrospective study plan has been carried out in 11 Coronary Care Units (CCU), in tertiary of Punjab, Pakistan. Information was derived from administrative hospital discharge data of AMI patients from 1 January 2016 to 31 December 2017 by three trained bio-statisticians. This study chooses the big cities of Punjab, such as Faisalabad, Lahore, Multan, Rawalpindi, and Islamabad, to measure the quality of care and its impact on in-hospital outcomes among AMI patients who have a strong economic background. The details of geographical, racial and economic factors of Punjab cities are available at the Pakistan Bureau of Statistics [19]. The study population consisted of patients who presented to emergency room within $24 \mathrm{~h}$ of the onset of an ischemic syndrome, and the ultimate primary diagnosis was AMI labeled as either STEMI or NSTEMI. Variables include patient demographics, clinical presentation, medical history, cardiovascular risk factors, co-morbidities, timings of care 
conveyance; treatments advised as well as major contraindications related to treatments, post-hospital discharge therapy, and in-hospital patient outcomes.

For hospitals with more prominent than 300 AMI cases, we excluded those that had no patient's outcome(death/survival). Thus, we were left with only 300 cases. For hospitals fewer than 300 AMI cases, all were chosen. Patients were excluded in the final analysis on the off chance that they were hospitalized for more than 90 days, in the event that they were transferred from another acute care facility, in the case they had stayed $<1$ day and discharged alive, and those with had lost information. After avoidance, a total of 2663 AMI cases-1454 STEMI and 1209 NSTEMI, were cleared out for final investigations. Ethical approval for the study was obtained from Ethics Review Committee of all hospital regulatory body Ministry of health Pakistan approval reference (KIIT 2019/PK 2019-25- MS 65).

\subsection{Quality Indicators}

Quality of care for AMI patients was investigated using 13 QIs at the primary and secondary levels. The relationship between adherence to these indicators and in-hospital outcomes was evaluated. The indicators of quality care measures included were the in-hospital advise of aspirin (QI1), beta blockers (QI2), clopidogrel (QI3), thrombolytic (QI4), electrocardiograph (ECG) (QI5), left ventricular function(LVF) assessment (QI6), coronary angiography (QI7), primary percutaneous coronary intervention(PCI) (QI8) and at secondary level, use of aspirin (QI9), beta-blocker (QI10), Angiotensin-Converting Enzyme (ACE) Inhibitor (QI11), Statin (QI12), Clopidogrel (QI13) at post-discharge. These measures are proposed by the Chinese health care system based upon the Modified Delphi process [20]. Unlike the indicators proposed by the American College of Cardiology and the American Heart Association (ACC/AHA), which consisted of only quality of care measures [21], The present investigation included additional structural and outcome indicators, based on the theoretical structure of Donabedian medical quality system [20-23]. Due to many differences between Pakistan and other developed countries when comparing health-care systems and how diseases are treated, we focused on using Chinese quality indicators for AMI care as both are developing countries. In-hospital mortality and length of stay (LOS) were considered as outcome measures.

\subsection{Statistical Analysis}

Baseline demographic characteristics of patients and co-morbidities for the selected population were summed up in the form of frequencies and percentages (Tables 1 and 2). In addition, for each baseline group, in-hospital mortality rate and LOS were also calculated as the outcome might also be influenced by patient's characteristics. We conducted a univariate analysis to examine the effects of patient characteristics and comorbidities on patient outcomes. Each QI was an independent variable separately and in-hospital outcome (mortality/LOS) was the dependent variable. The adherence rate was calculated for each QI as the sum of patients receiving care (numerator) divided by the number of patients meeting eligibility criteria (denominator) [20]. We calculated the patient composite score using the sum of numerator divided by the sum of denominator of all QIs. With patient-level and hospital-level risk factors as fixed effects and a random intercept for hospitals, unadjusted and adjusted hierarchical generalized linear model (HGLM) were used to investigate the impact of receiving treatment (usage of a single indicator/patient quality of care composite score) on in-hospital outcomes. For each QI, patients meeting the eligibility criteria were included in the analysis. The composite score was added as a continuous predictor variable, and regression coefficients were reported per $10 \%$ increment in composite score. All significant $(p<0.05)$ risks factors (listed in Tables 1 and 2$)$ in univariate analysis for patient characteristics were co-variables in the HGLM. Analysis of the abstracted data was performed using SAS version 9.3 (SAS Institute Inc., Cary, NC, USA). 


\section{Results}

\subsection{Clinical Characteristics}

Table 1 indicates the baseline and clinical attributes of admissions for AMI. Among 2663 patients, 1859 (69.81\%) of the admissions were male patients. In-hospital mortality rate accounted approximately same for both males and females as $8.55 \%$ and $8.58 \%$, respectively. An aggregate of 1197 patients was $>70$ years old with a higher death rate of $9.11 \% .2314$ patients were reported as obese, with a mortality rate of $8.69 \%$. Only $38 \%$ had health insurance. $56.37 \%$ were smokers and demonstrated a slightly higher rate of in-hospital mortality, i.e. $8.86 \%$ than those without smoking, i.e. $8.18 \%$. Approximately, half of them had a family history of ischemic heart disease (42.32\%). Among 2663 patients, 1052 had a background marked by hypertension and 909 had a history of diabetes mellitus. A number of STEMI admissions was more prominent than NSTEMI. However, the mortality rate was reported higher for NSTEMI admission. Similarly, patients admitted in generalized hospitals were significantly higher than the specialized hospital type, though specialized hospitals showed $9.96 \%$ mortality rate higher than the generalized one. The in-hospital mortality rate was $8.6 \%$ for our data sample. The mean and standard deviation of LOS was observed significantly associated with patient characteristics including age (for all group), obese (yes/no), insurance (Govt/private), prior myocardial infarction/coronary artery disease (MI/CAD: had/no), history of hypertension (had/no), type of MI (STEMI/NSTEMI) and hospital type (specialized/generalized). Table 2 illustrates the detailed statistics of comorbidities associated with in-hospital mortality and length of stay, also presented in Figure 1. Our findings show that patients with rheumatic heart disease and cerebrovascular disease at time of admission were observed significantly associated with in-hospital mortality. On the other hand, patients conceded with cardiogenic shock, higher level of lipids and thyroid as a comorbid factor were more likely to correlate with an increase in LOS.

Table 1. Univariate relationship between patient characteristics and outcome measures.

\begin{tabular}{|c|c|c|c|c|c|}
\hline \multirow{2}{*}{ Patient Characteristics } & \multirow{2}{*}{ Frequency (\%) } & \multicolumn{2}{|c|}{ In-Hospital Mortality } & \multicolumn{2}{|c|}{ LOS (day) } \\
\hline & & N (\%) & $p$ Value & mean $\pm S D$ & $p$ Value \\
\hline \multicolumn{6}{|l|}{ Age } \\
\hline$\sim 50$ & $316(11.87)$ & $26(8.23)$ & \multirow{4}{*}{0.8170} & $6.07 \pm 3.05$ & \multirow{4}{*}{$<0.0001$} \\
\hline $50-60$ & $414(15.55)$ & $32(7.73)$ & & $7.27 \pm 2.50$ & \\
\hline $60-70$ & $736(27.64)$ & $61(8.29)$ & & $7.64 \pm 2.29$ & \\
\hline $70 \sim$ & 1197 (44.95) & $109(9.11)$ & & $7.41 \pm 2.50$ & \\
\hline \multicolumn{6}{|l|}{ Sex } \\
\hline Male & $1859(69.81)$ & $159(8.55)$ & \multirow{2}{*}{0.9803} & $7.33 \pm 2.55$ & \multirow{2}{*}{0.6370} \\
\hline Female & $804(30.19)$ & $69(8.58)$ & & $7.29 \pm 2.59$ & \\
\hline \multicolumn{6}{|l|}{ Obese } \\
\hline No & $2314(86.89)$ & $201(8.69)$ & \multirow{2}{*}{0.5544} & $7.37 \pm 2.53$ & \multirow{2}{*}{0.0045} \\
\hline Yes & $349(13.11)$ & $27(7.74)$ & & $6.96 \pm 2.69$ & \\
\hline \multicolumn{6}{|l|}{ Insurance } \\
\hline Government & $673(25.27)$ & $60(8.92)$ & \multirow{3}{*}{0.9177} & $7.16 \pm 2.77$ & \multirow{3}{*}{0.0040} \\
\hline Private & $341(12.81)$ & $28(8.21)$ & & $7.49 \pm 2.78$ & \\
\hline Uninsured & 1649 (61.92) & $140(8.49)$ & & $7.70 \pm 2.46$ & \\
\hline \multicolumn{6}{|l|}{ Smoking } \\
\hline No & $1162(43.63)$ & $95(8.18)$ & \multirow{2}{*}{0.5308} & $7.32 \pm 2.57$ & \multirow{2}{*}{0.2555} \\
\hline Yes & $1501(56.37)$ & $133(8.86)$ & & $7.31 \pm 2.55$ & \\
\hline \multicolumn{6}{|l|}{ Family history of IHD * } \\
\hline No & $1536(57.68)$ & $132(8.59)$ & \multirow{2}{*}{0.9451} & $7.24 \pm 2.58$ & \multirow{2}{*}{0.1109} \\
\hline Yes & 1127 (42.32) & $96(8.52)$ & & $7.42 \pm 2.53$ & \\
\hline \multicolumn{6}{|l|}{ Prior MI/CAD } \\
\hline No & 1693 (63.57) & $153(9.04)$ & \multirow{2}{*}{0.2467} & $7.23 \pm 2.58$ & \multirow{2}{*}{0.0214} \\
\hline Yes & $970(36.43)$ & 75 (7.73) & & $7.46 \pm 2.58$ & \\
\hline
\end{tabular}


Table 1. Cont.

\begin{tabular}{|c|c|c|c|c|c|}
\hline \multirow{2}{*}{ Patient Characteristics } & \multirow{2}{*}{ Frequency (\%) } & \multicolumn{2}{|c|}{ In-Hospital Mortality } & \multicolumn{2}{|c|}{ LOS (day) } \\
\hline & & N (\%) & $p$ Value & mean $\pm S D$ & $p$ Value \\
\hline \multicolumn{6}{|l|}{ History Of DM } \\
\hline No & 1754 (65.87) & $151(8.61)$ & \multirow{2}{*}{0.9039} & $7.26 \pm 2.62$ & \multirow{2}{*}{0.2349} \\
\hline Yes & $909(34.13)$ & $77(8.47)$ & & $7.41 \pm 2.42$ & \\
\hline \multicolumn{6}{|l|}{ History of Hypertension } \\
\hline No & $1611(60.50)$ & $130(8.07)$ & \multirow{2}{*}{0.2612} & $7.20 \pm 2.40$ & \multirow[b]{2}{*}{0.0151} \\
\hline Yes & $1052(39.50)$ & $98(9.32)$ & & $7.48 \pm 2.48$ & \\
\hline \multicolumn{6}{|l|}{ MI } \\
\hline NSTEMI & $1209(45.40)$ & $124(10.26)$ & \multirow{2}{*}{0.0044} & $6.09 \pm 2.65$ & \multirow{2}{*}{$<0.0001$} \\
\hline STEMI & $1454(54.60)$ & $104(7.15)$ & & $8.33 \pm 1.96$ & \\
\hline \multicolumn{6}{|l|}{ Hospital type } \\
\hline Specialized & $1034(38.83)$ & $103(9.96)$ & \multirow{2}{*}{0.0397} & $7.08 \pm 2.64$ & \multirow{2}{*}{$<0.0001$} \\
\hline Generalized & 1629 (61.17) & 125 (7.67) & & $7.46 \pm 2.49$ & \\
\hline
\end{tabular}

* There is 1 missing value. Abbreviation: LOS, length of stay; SD, standard deviation; MI, Myocardial Infarction; IHD, Ischemic Heart Disease; DM, Diabetes Mellitus; CAD, Coronary Artery Disease; STEMI, ST-elevation myocardial infarction; NSTEMI, Non-ST-elevation myocardial infarction.

Table 2. Univariate analysis of comorbidities with in-hospital mortality and length of stay.

\begin{tabular}{|c|c|c|c|c|c|}
\hline \multirow{2}{*}{ Comorbidities } & \multirow{2}{*}{ Frequency (\%) } & \multicolumn{2}{|c|}{ In-Hospital Mortality } & \multicolumn{2}{|c|}{ LOS (day) } \\
\hline & & N (\%) & $p$ Value & Mean \pm SD & $p$ Value \\
\hline \multicolumn{6}{|l|}{$\begin{array}{l}\text { Rheumatic } \\
\text { heart disease }\end{array}$} \\
\hline $\begin{array}{c}\text { no } \\
\text { yes }\end{array}$ & $\begin{array}{c}2655(99.70) \\
8(0.30)\end{array}$ & $\begin{array}{c}226(8.51) \\
2(25.00)\end{array}$ & 0.0002 & $\begin{array}{l}7.32 \pm 2.56 \\
6.88 \pm 3.18\end{array}$ & 0.8367 \\
\hline \multicolumn{6}{|l|}{ Heart Failure } \\
\hline $\begin{array}{c}\text { no } \\
\text { yes }\end{array}$ & $\begin{array}{c}2615(98.20) \\
48(1.80)\end{array}$ & $\begin{array}{c}222(8.49) \\
6(12.50)\end{array}$ & 0.3251 & $\begin{array}{l}7.31 \pm 2.56 \\
7.35 \pm 2.37\end{array}$ & 0.9827 \\
\hline \multicolumn{6}{|l|}{ Cardiogenic Shock } \\
\hline $\begin{array}{c}\text { no } \\
\text { yes }\end{array}$ & $\begin{array}{l}2392(89.82) \\
271(10.18)\end{array}$ & $\begin{array}{l}204(8.53) \\
24(8.86)\end{array}$ & 0.8550 & $\begin{array}{l}7.27 \pm 2.57 \\
7.68 \pm 2.43\end{array}$ & 0.0137 \\
\hline \multicolumn{6}{|l|}{ Hypertension } \\
\hline $\begin{array}{c}\text { no } \\
\text { yes }\end{array}$ & $\begin{array}{c}1998(75.03) \\
665(24.97)\end{array}$ & $\begin{array}{l}167(8.36) \\
61(9.17)\end{array}$ & 0.5155 & $\begin{array}{l}7.30 \pm 2.57 \\
7.35 \pm 2.53\end{array}$ & 0.8212 \\
\hline \multicolumn{6}{|l|}{ Cerebrovascular disease } \\
\hline $\begin{array}{c}\text { no } \\
\text { yes }\end{array}$ & $\begin{array}{c}2253(84.60) \\
410(15.40)\end{array}$ & $\begin{array}{l}205(9.10) \\
23(5.61)\end{array}$ & 0.0022 & $\begin{array}{l}7.29 \pm 2.60 \\
7.43 \pm 2.32\end{array}$ & 0.5060 \\
\hline \multicolumn{6}{|l|}{ Gastrointestinal disease } \\
\hline $\begin{array}{l}\text { no } \\
\text { yes }\end{array}$ & $\begin{array}{c}2620(98.39) \\
43(1.61)\end{array}$ & $\begin{array}{l}226(8.63) \\
2(4.65)\end{array}$ & 0.3555 & $\begin{array}{l}7.32 \pm 2.56 \\
7.05 \pm 2.54\end{array}$ & 0.5060 \\
\hline \multicolumn{6}{|l|}{ Type-I DM } \\
\hline $\begin{array}{l}\text { no } \\
\text { yes }\end{array}$ & $\begin{array}{c}2307(86.63) \\
356(13.37)\end{array}$ & $\begin{array}{c}200(8.67) \\
28(7.87)\end{array}$ & 0.6138 & $\begin{array}{l}7.29 \pm 2.60 \\
7.48 \pm 2.26\end{array}$ & 0.5357 \\
\hline \multicolumn{6}{|l|}{ Type-II DM } \\
\hline $\begin{array}{c}\text { no } \\
\text { yes }\end{array}$ & $\begin{array}{c}2322(87.19) \\
341(12.81)\end{array}$ & $\begin{array}{l}197(8.48) \\
31(9.09)\end{array}$ & 0.7084 & $\begin{array}{l}7.33 \pm 2.56 \\
7.23 \pm 2.52\end{array}$ & 0.4519 \\
\hline \multicolumn{6}{|l|}{ Renal failure } \\
\hline $\begin{array}{l}\text { no } \\
\text { yes }\end{array}$ & $\begin{array}{l}2451(92.04) \\
212(7.96)\end{array}$ & $\begin{array}{l}206(8.40) \\
22(10.38)\end{array}$ & 0.3247 & $\begin{array}{l}7.34 \pm 2.54 \\
7.00 \pm 2.71\end{array}$ & 0.3972 \\
\hline \multicolumn{6}{|l|}{ Dysrhythmia } \\
\hline $\begin{array}{l}\text { no } \\
\text { yes }\end{array}$ & $\begin{array}{c}2485(93.32) \\
178(6.68)\end{array}$ & $\begin{array}{c}216(8.69) \\
12(6.74)\end{array}$ & 0.3689 & $\begin{array}{l}7.29 \pm 2.57 \\
7.66 \pm 2.69\end{array}$ & 0.1766 \\
\hline \multicolumn{6}{|c|}{ Peripheral Vascular Disease } \\
\hline $\begin{array}{l}\text { no } \\
\text { yes }\end{array}$ & $\begin{array}{l}2389(89.71) \\
274(10.29)\end{array}$ & $\begin{array}{l}201(8.41) \\
27(9.85)\end{array}$ & 0.4196 & $\begin{array}{l}7.30 \pm 2.58 \\
7.41 \pm 2.32\end{array}$ & 0.9493 \\
\hline
\end{tabular}


Table 2. Cont.

\begin{tabular}{|c|c|c|c|c|c|}
\hline \multirow{2}{*}{ Comorbidities } & \multirow{2}{*}{ Frequency (\%) } & \multicolumn{2}{|c|}{ In-Hospital Mortality } & \multicolumn{2}{|c|}{ LOS (day) } \\
\hline & & N (\%) & $p$ Value & Mean \pm SD & $p$ Value \\
\hline \multicolumn{6}{|l|}{ COPD } \\
\hline no & $2525(94.82)$ & $215(8.51)$ & \multirow{2}{*}{0.7113} & $7.30 \pm 2.56$ & \multirow{2}{*}{0.1561} \\
\hline yes & $138(5.18)$ & $13(9.42)$ & & $7.61 \pm 2.43$ & \\
\hline \multicolumn{6}{|l|}{ Liver disease } \\
\hline no & $2615(98.20)$ & $224(8.57)$ & \multirow{2}{*}{0.9545} & $7.31 \pm 2.56$ & \multirow{2}{*}{0.4408} \\
\hline yes & $48(1.80)$ & $4(8.33)$ & & $7.60 \pm 2.44$ & \\
\hline \multicolumn{6}{|l|}{ Hypercholesterolemia } \\
\hline no & $1614(60.61)$ & $140(8.67)$ & \multirow[b]{2}{*}{0.7972} & $7.22 \pm 2.59$ & \multirow[b]{2}{*}{0.0499} \\
\hline yes & 1049 & $88(8.39)$ & & $7.46 \pm 2.50$ & \\
\hline \multicolumn{6}{|l|}{ Thyroid Disorder } \\
\hline no & 1950 & $175(8.97)$ & \multirow{2}{*}{0.2082} & $7.19 \pm 2.62$ & \multirow{2}{*}{$<0.0001$} \\
\hline yes & 713 & $53(7.43)$ & & $7.65 \pm 2.34$ & \\
\hline
\end{tabular}

Abbreviation: LOS, length of stay; SD, standard deviation; COPD, chronic obstructive pulmonary disease; DM, diabetes mellitus.

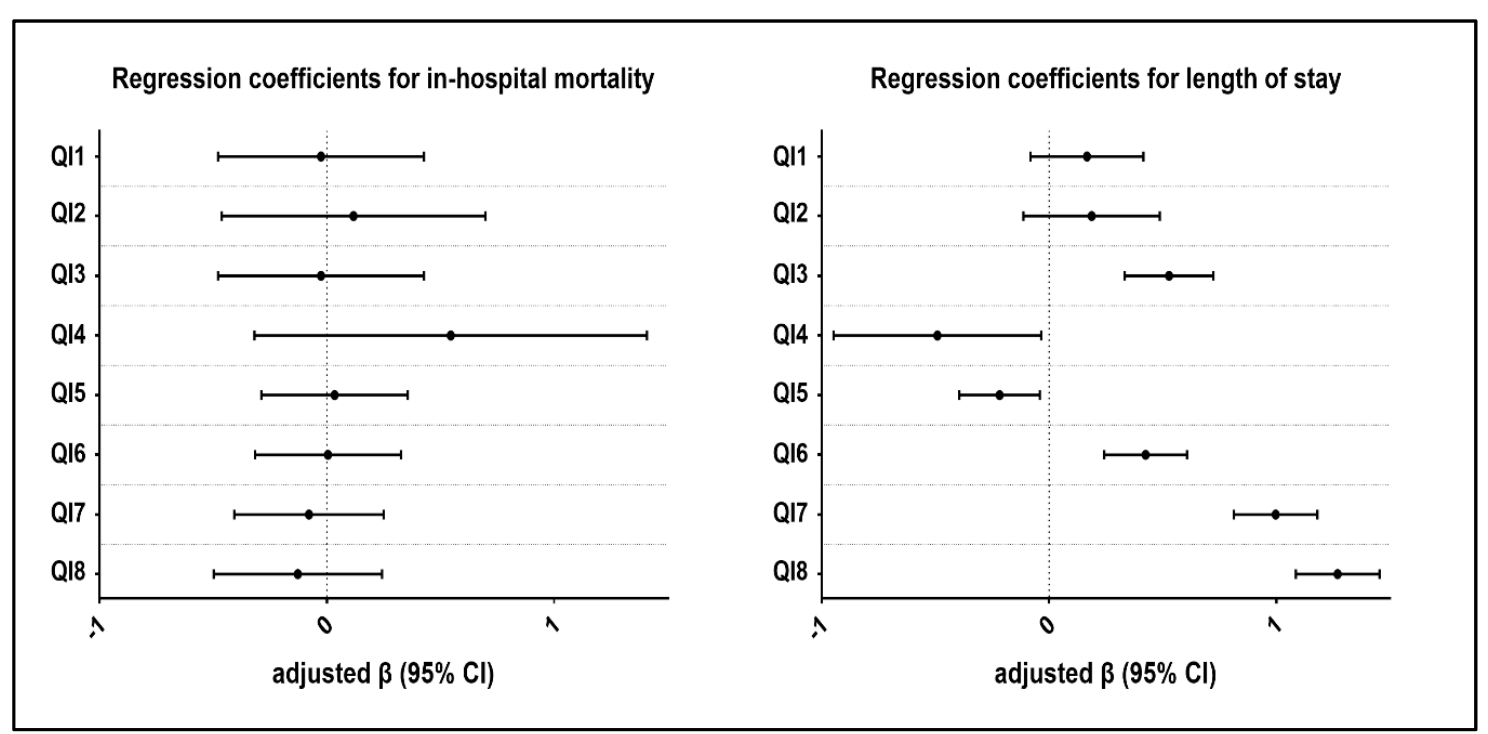

Figure 1. Regression coefficients estimation for in-house mortality and length of stay.

\subsection{Compliance Measurements}

At a primary level of care,1132 of 1549 patients (73.08\%) with AMI furnished with aspirin within three hours of hospital arrival. 785 patients were eligible for receiving beta-blockers, only $306(38.98 \%)$ actually received it. An aggregate of 1634 of 2408 patients (67.86\%) was facilitated with clopidogrel. Thrombolytic appeared with the lowest compliance rate of $7.52 \%$. ECG, left ventricular ejection fraction (LVEF) assessment, and coronary angiography were recommended to every chosen patient however just 1664 (62.49\%), 1672 (62.79\%), and 1767 (66.35\%) patients were given the exhorted therapy, individually. Of 2366 patients who were considered as eligible for percutaneous coronary intervention (PCI) treatment, only 884 (37.36\%) acquired it. At the secondary dimension of consideration, aspirin appeared with a compliance rate of $47.97 \%$, beta-blocker with $22.29 \%$, clopidogrel with $45.35 \%$, statin with $36.39 \%$, and ACE inhibitor with $38.02 \%$. Among all indicators (at the primary and secondary level of care), the most noteworthy compliance rate was of aspirin whereas, thrombolytic showed the least rate (Table 3). 
Table 3. Adherence to performance measures.

\begin{tabular}{lccc}
\hline Quality Indicators (QI) & $\begin{array}{c}\text { Eligible Patients, } \\
\text { No. (\%) }\end{array}$ & $\begin{array}{c}\text { Overall } \\
\text { Adherence, \% }\end{array}$ & $\begin{array}{c}\text { Hospital Variation, } \\
\text { Range (\%) }\end{array}$ \\
\hline $\begin{array}{l}\text { QI1: Aspirin prescribed within 3h of } \\
\text { hospital arrival }\end{array}$ & $1549(58.17)$ & 73.08 & $62.11-85.81$ \\
QI2: Beta-blockers within 12h of arrival & $785(23.69)$ & 38.98 & $32.89-45.45$ \\
QI3: Clopidogrel within 12h of arrival & $2408(90.42)$ & 67.86 & $62.68-73.27$ \\
QI4: Thrombolytics received within 30 min & $971(36.46)$ & 7.52 & $3.57-11.43$ \\
of hospital arrival & $2663(100.00)$ & 62.49 & $57.74-71.84$ \\
QI5: ECG within 10 min of hospital arrival & $2663(100.00)$ & 62.79 & $56.33-67.53$ \\
QI6: Left ventricular function assessment & $2663(100.00)$ & 66.35 & $60.85-71.78$ \\
QI7: Coronary angiography performed & $2366(88.85)$ & 37.36 & $29.52-43.59$ \\
during hospital stay & $1549(58.17)$ & 47.97 & $38.51-61.29$ \\
QI8: Primary PCI & $785(29.48)$ & 22.29 & $10.94-34.21$ \\
Prescription filled post-discharge & $2408(90.42)$ & 45.35 & $35.36-53.59$ \\
QI9: Aspirin & $2020(75.85)$ & 36.39 & $30.11-41.76$ \\
QI10: Beta-blocker & $2567(96.40)$ & 38.02 & $29.70-45.54$ \\
QI11: Clopidogrel & & & \\
QI12: Statin & $2663(100.00)$ & 8.56 & $5.56-12.50$ \\
QI13: ACE inhibitor & & & \\
Outcome indicators & In-hospital mortality &
\end{tabular}

Abbreviation: PCI, percutaneous coronary intervention. ${ }^{a}$ Eligible patients are those with definite indications but no documented contraindications.

Aspirin and clopidogrel being pharmacological indicators showed better conformance at hospital level of care whereas in the case of thrombolytic poorer adherence was observed. For PCI, as an invasive procedure, there was no significant conformance observed across hospitals. At secondary level of prevention, hospital adherence was slightly on the rise but yet not satisfactory (Table 3). The median (Interquartile range) composite score for patients was $0.50(0.38-0.63)$.

\subsection{Association between Process Indicators and Outcomes}

Table 4 summarizes the results of adjusted and unadjusted regression coefficients with $95 \%$ confidence interval for the association between process measures and patient outcome at discharge. 3 out of 8 QIs showed a negative association on in-hospital mortality, although insignificant, which indicates that patients who received aspirin, coronary angiography, and PCI during their hospital stay were at lower risk of dying in the hospital. After adjustment for confounding factors, the results remain unchanged, and no significant differences were observed. In contrast, 4 out of 8 QIs showed strong positive and significant association regarding LOS, which suggested that AMI patients who received clopidogrel, LVEF assessment, coronary angiography, and PCI during their hospital stay were more likely to be associated with an increased LOS. Overall PCI, turned out to be the largest risk factor on account of increased LOS whereas aspirin, beta-blockers and thrombolytic were not considered as predictive indicators.

Table 5 summarizes the impact of the patient composite scores on in-hospital mortality. Overall, the patient composite scores were observed insignificantly associated with in-hospital mortality $(p>0.05)$, although only one measure, i.e., STEMI vs. NSTEMI gives a statistically significant relationship at $p=0.05$ level. However, at a more aggregate degree, overall composite score (per 10\%) was insignificantly but positively associated with in-hospital deaths. The factor with the greatest weight affecting in-hospital mortality is rheumatic heart disease, although found insignificant. On the contrary, the effect of patient composite score on LOS gives a little different result. The effect of patient composite score on LOS for all age groups was found significantly and positively associated at $p=0.05$ level of significance.

Interestingly, the effect of patient composite score on length of stay for all age groups was found significantly and positively associated at $p=0.05$ level of significance. Also, STEMI vs. NSTEMI and 
thyroid patients ended up being statistically significant, with a positive association towards length of stay. More precisely, overall composite score (per10\%) was observed statistically significant giving an indication of meaningful addition to our model (Table 6). The median (Interquartile range) composite score for patients was $0.50(0.38-0.63)$. As can be seen from these two tables that STEMI vs. NSTEMI is the common factor of patient composite score which is associated with in-hospital mortality and LOS. The overall low quality of patient care was observed based on composite score.

Table 4. Association of process measures with clinical outcomes among acute myocardial infarction patients.

\begin{tabular}{ccccc}
\hline $\begin{array}{c}\text { Quality } \\
\text { Indicators }\end{array}$ & \multicolumn{2}{c}{ In-Hospital Mortality } & \multicolumn{2}{c}{ Length of Stay } \\
\cline { 2 - 5 } & $\begin{array}{c}\text { Adjusted } \beta \\
(\boldsymbol{p} \text { Value })\end{array}$ & $\begin{array}{c}\text { Unadjusted } \boldsymbol{\beta} \\
(\boldsymbol{p} \text { Value })\end{array}$ & $\begin{array}{c}\text { Adjusted } \boldsymbol{\beta} \\
(\boldsymbol{p} \text { Value })\end{array}$ & $\begin{array}{c}\text { Unadjusted } \beta \\
(\boldsymbol{p} \text { Value })\end{array}$ \\
\hline QI1 & $-0.0258(0.9014)$ & $-0.0382(0.8540)$ & $0.1668(0.1880)$ & $0.2930(0.0340)$ \\
QI2 & $0.1174(0.6616)$ & $0.1079(0.6856)$ & $0.1873(0.2207)$ & $0.2337(0.1456)$ \\
QI3 & $0.0238(0.8831)$ & $0.0156(0.9229)$ & $0.5278(<0.0001)$ & $0.7994(<0.0001)$ \\
QI4 & $0.5451(0.1898)$ & $0.6093(0.1457)$ & $-0.4913(0.0351)$ & $-0.4994(0.0328)$ \\
Q15 & $0.0342(0.8177)$ & $0.0525(0.7229)$ & $-0.2179(0.0159)$ & $-0.2913(0.0043)$ \\
QI6 & $0.0048(0.9742)$ & $-0.0636(0.6647)$ & $0.4251(<0.0001)$ & $0.8413(<0.0001)$ \\
QI7 & $-0.0786(0.6057)$ & $-0.1535(0.3110)$ & $0.9974(<0.0001)$ & $1.5171(<0.0001)$ \\
QI8 & $-0.1276(0.4603)$ & $-0.2174(0.2102)$ & $1.2699(<0.0001)$ & $1.7515(<0.0001)$ \\
\hline
\end{tabular}

* The definition of quality indicators (QIs) please refer to Table 3. All significant $(p<0.10)$ risks factors (listed in Table 1) in univariate analysis for patient characters were included in the hierarchical generalized linear model.

Table 5. Effect of patient composite scores on in-hospital mortality.

\begin{tabular}{ccc}
\hline Factors & Regression Coefficient, 95\%CI & $p$ Value \\
\hline STEMI vs. NSTEMI & $1.451(1.026,2.053)$ & 0.0379 \\
Generalized vs. Specialized & $0.877(0.613,1.254)$ & 0.4270 \\
Hospital & $0.620(0.355,1.082)$ & \\
Cerebra disease & $2.346(0.070,78.121)$ & 0.0849 \\
Rheumatic Heart Disease (RHD) & $1.003(0.937,1.073)$ & 0.4952 \\
Composite score (per 10\%) & 0.9284 \\
\hline
\end{tabular}

The composite score was added as a continuous predictor variable, and regression coefficients were reported per $10 \%$ increment in composite score. Abbreviation: STEMI, ST-elevation myocardial infarction; NSTEMI, Non-ST-elevation myocardial infarction.

Table 6. Effect of patient composite scores on length of stay.

\begin{tabular}{|c|c|c|}
\hline Factors & Regression Coefficient, $95 \% \mathrm{CI}$ & $p$ Value \\
\hline \multicolumn{3}{|l|}{ Age } \\
\hline $50-60$ vs. $\sim 50$ & $0.481(0.132,0.829)$ & 0.0069 \\
\hline $60-70$ vs. $\sim 50$ & $0.751(0.432,1.071)$ & $<0.0001$ \\
\hline $70 \sim$ vs. $\sim 50$ & $0.587(0.282,0.891)$ & 0.0002 \\
\hline \multicolumn{3}{|l|}{ Insurance } \\
\hline Government vs. Uninsured & $0.147(-0.059,0.354)$ & 0.1623 \\
\hline Private vs. Uninsured & $0.202(-0.069,0.474)$ & 0.1443 \\
\hline Prior MI/CAD & $0.021(-0.210,0.251)$ & 0.8595 \\
\hline History of Hypertension & $0.137(-0.091,0.365)$ & 0.2401 \\
\hline STEMI vs. NSTEMI & $2.049(1.871,2.227)$ & $<0.0001$ \\
\hline Generalized vs. Specialized hospital & $0.168(-0.164,0.499)$ & 0.3213 \\
\hline thyroid & $0.257(0.058,0.455)$ & 0.011 \\
\hline lipids & $0.179(-0.001,0.359)$ & 0.051 \\
\hline Composite score (per 10\%) & $0.258(0.217,0.299)$ & $<0.0001$ \\
\hline
\end{tabular}

The composite score was added as a continuous predictor variable, and regression coefficients were reported per $10 \%$ increment in composite score. Abbreviation: MI, Myocardial Infarction; CAD, Coronary Artery Disease; STEMI, ST-elevation myocardial infarction; NSTEMI, Non-ST-elevation myocardial infarction. 


\section{Discussion}

This study provides the most recent statistics for evaluating quality of care delivery for patients with acute MI in Pakistan. A well-prepared checklist was administered in order to get the characteristics of AMI patients. Further, this study has observed overall a low compliance rate, which is explicitly lower than the international standards among AMI patients (STEMI and NSTEMI). No significant association was observed between QIs and in-hospital mortality. 4 out of 8 quality indicators showed strong positive and significant association in terms of LOS. Likewise, patient composite score was also observed significantly associated with LOS. Composite performance measures are often attached to regulatory mechanisms whereby hospitals are rewarded or punished according to the outcome of the composite indicator. The use and publication of composite performance measures can generate both positive and negative behavioral responses [24].

Except for aspirin, other indicators all demonstrated poor compliance rates. This finding is inconsistent with a study from China that reported weak conformance for a few indicators [25]. The possible reasons for low compliance rate could be the lack of staff and resources at governmental hospitals. Lack of AMI quality improvement programs at hospital levels may be another reason for poor performance in AMI care management. Studies have suggested that AMI patients face significant delays before adequate treatment is available to them. Framework delays and access to reperfusion are distinguished as drivers of destitute compliance rate and remained significant challenges inside the AMI framework of care within the hospitals of Pakistan $[15,26]$. Execution in quality indicators measurement can, subsequently, be progressed by simply expanding adherence to these guidelines. However, the results indicate that serious attention is required to improve the steps of quality of care over 11 hospitals.

Every single indicator must be characterized with the end goal of assessment. Few indicators are time confined, while others such as coronary angiography and PCI are free from time restriction [11,27]. Both procedures cost high and most of the eligible patients can't manage the cost of it. In any case, for a clear reason, it is still recommended as procedure of care indicator. The compliance rate should way to deal with $100 \%$ when every single qualified patient gets exhorted medication or therapy.

Nonetheless, because occasionally contraindications to advised therapy are not constantly enrolled, the recommended benchmark esteems underneath 100\% [28]. It likewise indicates that there is still opportunity to get better notwithstanding the absence of consistency with guidelines. We found that in univariate analysis the effect of patient's characteristics on LOS is more significant than in-hospital mortality. Also, in multivariate analyses adherence to QIs was found significantly correlated with LOS. Postoperative complications or comorbidities associated at time of admissions may be a reason for increased LOS.

Another finding of this study is the concept of a wide scope of comorbidities associated with the time of admission and their impact on clinical outcomes. The measurement of comorbidities remains an area of concern for scientists and clinicians [29]. Studies have shown that prolonged hospitalization, decreased quality of life, and increased health-care costs can be affected by comorbidities associated at the time of admissions for patients with AMI [30-32]. The main and foremost limitation of our study was inaccessibility of long-term outcome of the selected sampled population. As a result of no subsequent system in Pakistan, it was not in any way conceivable to induce data of these patients after discharge. Therefore, in comparison to others, this study only comprises of patient data during their hospital stay. Another impediment of this study was precision in numerators and denominators.

We believe these results give a credible source of valid information when assessing and improving the quality of medical care. Moreover, this source of information will allow an easier comparison, both within and between countries; this, in turn, leads to more accurate and trustworthy reporting to drive further assessment and improvement in quality of care and outcomes. Clinical indicators function as the spine for assessing the quality of care provided across the full spectrum of domains of patient care [33]. Therefore, monitoring the health care quality framework, these clinical measures assume 
an essential job. To ensure their unwavering quality and legitimacy, they should be developed and implemented with transparency.

The therapeutic records of patients in Pakistani hospitals are intended for administrative instead of evaluation purposes, which may have resulted in some evidence being missed or inaccurate. A few patients denied a recommended therapy or left against medical advice on account of their poor budgetary circumstances, so the numerator could be thought little off. Some data about contraindications may be erroneously recorded, causing an overestimation of the denominator. Besides, uncertain affiliations of value measures on healthcare procedures and mortality could likewise be clarified by non-medical care-related variables, e.g., financial status and local healthcare organizations that would influence the health outcomes after discharge. These variables were out of the control of the hospital and will exclusively affect the mortality proportion. Moreover, quality of care measurement depends on the detectability of information gathered in medicinal records and may not precisely reflect care conveyance.

\section{Conclusions}

This study in a resource-limited setting, showed some disparities between guidelines and clinical practice. Low adherence to performance measures strongly compel to focus on guideline-based tools for AMI in Pakistan. Taking everything into account, our analyses of the quality of care indicators for AMI patients reveal less satisfactory outcomes. We prescribe a more grounded spotlight on Pakistani national health services regulation and coordination in AMI care and the foundation of national benchmarks for AMI-explicit consideration thrombolytic and PCI related quality indicators to encourage the nature of consideration for AMI patients in Pakistan. Further investigations should be led in other provinces of Pakistan to distinguish, evaluate, and improve the AMI care. Besides, we recommend that clinicians and health care providers use both quality indicators and outcome measures to optimally assess overall clinic quality of care for AMI. The difference in treatment quality between hospitals shows that there is room for improvement in hospitals with poor quality, and it can also help hospitals to make targeted improvements.

Author Contributions: S.R. had full access to all the data in the study and takes responsibility for the integrity of the data and the accuracy of the data analysis. Study concept and design: S.R., X.L., C.W., M.I. Acquisition, analysis, or interpretation of data: All authors. Drafting of the manuscript: S.R., M.I. and E.R. Critical revision of the manuscript for important intellectual content: All authors. Statistical analysis: S.R., X.L., M.I, E.R. Obtained funding: M.L. Study supervision: M.L.

Funding: This work was supported by National Natural Science Foundation of China [81573255 to Meina Liu].

Conflicts of Interest: The authors declare no conflict of interest.

\section{References}

1. Califf, R.M.; Peterson, E.D.; Gibbons, R.J.; Garson, A.; Brindis, R.G.; Beller, G.A.; Smith, S.C. Integrating quality into the cycle of therapeutic development. J. Am. Coll. Cardiol. 2002, 40, 1895-1901. [CrossRef]

2. Schiele, F.; Gale, C.P.; Simon, T.; Fox, K.A.A.; Bueno, H.; Lettino, M.; Tubaro, M.; Puymirat, E.; Ferrières, J.; Meneveau, N.; et al. Assessment of Quality Indicators for Acute Myocardial Infarction in the FAST-MI (French Registry of Acute ST-Elevation or Non-ST-Elevation Myocardial Infarction) Registries. Circ. Cardiovasc. Qual. Outcomes 2017, 10, e003336. [CrossRef] [PubMed]

3. Kontos, M.C.; Rennyson, S.L.; Chen, A.Y.; Alexander, K.P.; Peterson, E.D.; Roe, M.T. The association of myocardial infarction process of care measures and in-hospital mortality: A report from the $\mathrm{NCDR}^{\circledR}$. Am. Heart J. 2014, 168, 766-775. [CrossRef] [PubMed]

4. Peterson, E.D.; Roe, M.T.; Mulgund, J.; DeLong, E.R.; Lytle, B.L.; Brindis, R.G.; Smith, S.C.; Pollack, C.V.; Newby, L.K.; Harrington, R.A.; et al. Association Between Hospital Process Performance and Outcomes Among Patients With Acute Coronary Syndromes. JAMA 2006, 295, 1912. [CrossRef] [PubMed]

5. Araújo, C.; Laszczyńska, O.; Viana, M.; Dias, P.; Maciel, M.J.; Moreira, I.; Azevedo, A. Quality of Care and 30-day Mortality of Women and Men With Acute Myocardial Infarction. Rev. Esp. Cardiol. 2019, 72, 543-552. 
6. Baldwin, L.M.; MacLehose, R.F.; Hart, L.G.; Beaver, S.K.; Every, N.; Chan, L. Quality of Care for Acute Myocardial Infarction in Rural Urban US Hospitals. J. Rural Health 2004, 20, 99-108. [CrossRef]

7. Krumholz, H.M.; Anderson, J.L.; Bachelder, B.L.; Fesmire, F.M.; Fihn, S.D.; Foody, J.M.; Ho, P.M.; Kosiborod, M.N.; Masoudi, F.A.; Nallamothu, B.K. ACC/AHA 2008 Performance Measures for Adults With ST-Elevation and Non-ST-Elevation Myocardial Infarction. Circulation 2008, 118, 2596-2648. [CrossRef]

8. Chew, D.P.; Scott, I.A.; Cullen, L.; French, J.K.; Briffa, T.G.; Tideman, P.A.; Woodruffe, S.; Kerr, A.; Branagan, M.; Aylward, P.E.G. National Heart Foundation of Australia and Cardiac Society of Australia and New Zealand: Australian clinical guidelines for the management of acute coronary syndromes 2016. Med. J. Aust. 2016, 205, 128-133. [CrossRef]

9. Tu, J.V.; Khalid, L.; Donovan, L.R.; Ko, D.T. Indicators of quality of care for patients with acute myocardial infarction. Cmaj 2008, 179, 909-915. [CrossRef]

10. Ibanez, B.; James, S.; Agewall, S.; Antunes, M.J.; Bucciarelli-Ducci, C.; Bueno, H.; Caforio, A.L.P.; Crea, F.; Goudevenos, J.A.; Halvorsen, S.; et al. 2017 ESC Guidelines for the management of acute myocardial infarction in patients presenting with ST-segment elevation. Eur. Heart J. 2018, 39, 119-177. [CrossRef]

11. Task Force on the management of ST-segment elevation acute myocardial infarction of the European Society of Cardiology (ESC); Steg, P.G.; James, S.K.; Atar, D.; Badano, L.P.; Blömstrom-Lundqvist, C.; Borger, M.A.; Di Mario, C.; Dickstein, K.; Ducrocq, G.; et al. ESC Guidelines for the management of acute myocardial infarction in patients presenting with ST-segment elevation. Eur. Heart J. 2012, 33, 2569-2619. [CrossRef] [PubMed]

12. Jneid, H.; Addison, D.; Bhatt, D.L.; Fonarow, G.C.; Gokak, S.; Grady, K.L.; Green, L.A.; Heidenreich, P.A.; Ho, P.M.; Jurgens, C.Y.; et al. 2017 AHA/ACC Clinical Performance and Quality Measures for Adults With ST-Elevation and Non-ST-Elevation Myocardial Infarction: A Report of the American College of Cardiology/American Heart Association Task Force on Performance Measures. J. Am. Coll. Cardiol. 2017, 70, 2048-2090. [CrossRef] [PubMed]

13. Tran, D.T.; Welsh, R.C.; Ohinmaa, A.; Thanh, N.X.; Bagai, A.; Kaul, P. Quality of Acute Myocardial Infarction Care in Canada: A 10-Year Review of 30-Day In-Hospital Mortality and 30-Day Hospital Readmission. Can. J. Cardiol. 2017, 33, 1319-1326. [CrossRef]

14. Mozaffarian, D.; Benjamin, E.J.; Go, A.S.; Arnett, D.K.; Blaha, M.J.; Cushman, M.; Das, S.R.; De Ferranti, S.; Després, J.P.; Fullerton, H.J.; et al. Executive summary: Heart disease and stroke statistics-2016 update: A Report from the American Heart Association. Circulation 2016, 133, 447-454. [CrossRef] [PubMed]

15. Khursheed, M.; Fayyaz, J.; Feroze, A.; Shakeel, N.; Bhatti, J.A. Time to treatment in patients of suspected acute coronary syndrome in Pakistan: A clinical audit. Hear. Lung J. Acute Crit. Care 2015, 44, 63-67. [CrossRef] [PubMed]

16. Jafary, M.H.; Samad, A.; Ishaq, M.; Jawaid, S.A.; Ahmad, M.; Vohra, E.A. Profile of Acute Myocardial Infarction (AMI) in Pakistan. Pakistan J. Med. Sci. 2007, 23, 485-489.

17. Malik, S.M.; Bhutta, Z.A. Reform of primary health care in Pakistan. Lancet 2018, 392, 1375-1377. [CrossRef]

18. Kutz, A.; Gut, L.; Ebrahimi, F.; Wagner, U.; Schuetz, P.; Mueller, B. Association of the Swiss Diagnosis-Related Group Reimbursement System With Length of Stay, Mortality, and Readmission Rates in Hospitalized Adult Patients. JAMA Netw. Open 2019, 2, e188332. [CrossRef]

19. Statistics, P.B. of Pakistan Bureau of Statistics. Available online: http://www.pbs.gov.pk/publications.

20. Sun, H.; Liu, M.; Hou, S. Quality indicators for acute myocardial infarction care in China. Int. J. Qual. Health Care 2011, 23, 365-374. [CrossRef]

21. Nishimura, R.A.; Otto, C.M.; Bonow, R.O.; Carabello, B.A.; Erwin, J.P.; Guyton, R.A.; O'Gara, P.T.; Ruiz, C.E.; Skubas, N.J.; Sorajja, P.; et al. 2014 AHA/ACC guideline for the management of patients with valvular heart disease: Executive summary: A report of the american college of cardiology/american heart association task force on practice guidelines. Circulation 2014, 129, 2440-2492. [CrossRef]

22. Rublee, D.A. The Quality of Care: How Can It Be Assessed? JAMA J. Am. Med. Assoc. 1989, $261,1151$. [CrossRef]

23. Donabedian, A. Methods for deriving criteria for assessing the quality of medical care. Med. Care Rev. 1980, 37, 653. [PubMed]

24. Jacobs, R.; Goddard, M.; Smith, P.C. Composite Performance Measures in the Public Sector; Centre for Health Economics, University of York: York, UK, 2007; Volume 16. 
25. Wang, Y.; Fu, R.; Wang, Z.; Bao, H.; Chen, Y.; Yang, F.; Luo, X.; Liu, M. Assessing the Quality of Care for Patients with Acute Myocardial Infarction in China. Clin. Cardiol. 2015, 38, 327-332. [CrossRef] [PubMed]

26. Khan, M.S.; Jafary, F.H.; Faruqui, A.M.; Rasool, S.I.; Hatcher, J.; Chaturvedi, N.; Jafar, T.H. High prevalence of lack of knowledge of symptoms of acute myocardial infarction inPakistan and its contribution to delayed presentationto the hospital. BMC Public Health 2007, 7, 284. [CrossRef] [PubMed]

27. Ryan, T.J.; Antman, E.M.; Brooks, N.H.; Califf, R.M.; Hillis, L.D.; Hiratzka, L.F.; Rapaport, E.; Riegel, B.; Russell, R.O.; Smith, E.E. 1999 update: ACC/AHA guidelines for the management of patients with acute myocardial infarction: a report of the American College of Cardiology/American Heart Association Task Force on Practice Guidelines (Committee on Management of Acute Myocardial Infar. J. Am. Coll. Cardiol. 1999, 34, 890-911. [CrossRef]

28. Lee, D.S.; Tran, C.; Flintoft, V.; Grant, F.C.; Liu, P.P.; Tu, J.V. Canadian Cardiovascular Outcomes Research Team/Canadian Cardiovascular Society Heart Failure Quality Indicator Panel CCORT/CCS quality indicators for congestive heart failure care. Can. J. Cardiol. 2003, 19, 357-364.

29. Meghani, S.H.; Buck, H.G.; Dickson, V.V.; Hammer, M.J.; Rabelo-Silva, E.R.; Clark, R.; Naylor, M.D. The Conceptualization and Measurement of Comorbidity: A Review of the Interprofessional Discourse. Nurs. Res. Pract. 2013, 2013, 192782. [CrossRef]

30. Marengoni, A.; Angleman, S.; Melis, R.; Mangialasche, F.; Karp, A.; Garmen, A.; Meinow, B.; Fratiglioni, L. Aging with multimorbidity: A systematic review of the literature. Ageing Res. Rev. 2011, 10, 430-439. [CrossRef]

31. Lu, S.F.; Lu, L.X.; Smith, S.C.; Dai, X. Acute Myocardial Infarction in Patients with Paraplegia: Characteristics, Management, and Outcomes. Am. J. Med. 2018, 131, 574.e1-574.e11. [CrossRef]

32. Patel, R.S.; Shrestha, S.; Saeed, H.; Raveendranathan, S.; Isidahome, E.E.; Ravat, V.; Fakorede, M.O.; Patel, V. Comorbidities and Consequences in Hospitalized Heart Failure Patients with Depression. Cureus 2018, 10, e3193. [CrossRef]

33. Mainz, J. Developing evidence-based clinical indicators: a state of the art methods primer. Int. J. Qual. Health Care 2003, 15, i5-i11. [CrossRef] [PubMed] 